Melbourne, Australia and highlights $\mathrm{Mg}$ as an important sexually transmitted infection. Increase in detection of $\mathrm{Mg}$ in anal swabs also highlights the importance of rectal testing in symptomatic males.

Disclosure of interest statement No disclosure to declare.

\section{P07.02 EVALUATION OF THE HOLOGIC TRANSCRIPTION MEDIATED AMPLIFICATION ASSAY FOR DETECTION OF MYCOPLASMA GENITALIUM FROM URINE SAMPLES}

\footnotetext{
1,2,3 SN Tabrizi* ${ }^{2} \mathrm{AM}$ Costa, ${ }^{2} \mathrm{~S}$ Su, ${ }^{4} \mathrm{P}$ Lowe, ${ }^{5} \mathrm{CS}$ Bradshaw, ${ }^{5} \mathrm{CK}$ Fairley, $1,2,3 \mathrm{SM}$ Garland. ${ }^{1}$ Murdoch Childrens Research Institute; ${ }^{2}$ The Royal Children's and the Royal Women's Hospitals; ${ }^{3}$ University of Melbourne; ${ }^{4}$ Hologic, ${ }^{5}$ Melbourne Sexual Health Centre
}

\subsection{6/sextrans-2015-052270.318}

Introduction M. genitalium is an emerging sexually transmitted pathogen, with a strong association with urethritis, cervicitis and pelvic inflammatory disease. Detection of this bacterium by routine culture is not practical and routine diagnosis and screening for M. genitalium by molecular techniques has been hampered by lack of readily available commercial assays. A preliminary version of a commercial amplification assay is currently available on the Panther platform and was evaluated against an in-house qPCR assay currently in use for routine diagnostics.

Methods Overall 1000 consecutive urine samples from men and women were utilised for this evaluation. Over the course of 3 months, urine samples were obtained from consecutive symptomatic men and women being screened for M. genitalium at Melbourne Sexual Health Centre, as well as women being screened prior to termination of pregnancy at the Royal Women's Hospital, Melbourne. The primary Hologic assay targeting $80 \mathrm{bp}$ region of 16s rRNA was compared to the in-house diagnostic assay which targets a $517 \mathrm{bp}$ region of $16 \mathrm{~S}$ gene, as well as a second 16s rRNA target available on the Hologic platform.

Results The comparison of the two targets available on the Hologic platform showed very high correlation $(k=0.9795 \%$ CI $0.93-1.00)$. Comparison of primary Hologic assay to inhouse $16 \mathrm{~S}$ qPCR assay, also showed very good correlation $(\mathrm{K}=$ $0.8495 \%$ CI $0.75-0.93$ ). Overall, both primary and secondary Hologic assays on Panther were more sensitive than the $16 \mathrm{~S}$ qPCR for detection of M. genitalium in urine specimens.

Conclusion The M. genitalium assay on the Hologic platform integrated well with the laboratory procedures allowing rapid testing and possibility of rapid and accurate reporting using integration with laboratory information system. Overall the Hologic assay for detection of $M$. genitalium offers a simple, accurate and sensitive platform for diagnostic laboratories for detection of this important upcoming pathogen.

Disclosure of interest statement Hologic supplied the diagnostic kits and the Panther platform to conduct this study.

\section{P07.03 CLINICAL PERFORMANCE EVALUATION OF A NEW, RAPID POINT-OF-CARE SYSTEM FOR DETECTING CHLAMYDIA TRACHOMATIS}

${ }^{1} \mathrm{DM}$ Pearce*, ${ }^{2} \mathrm{~S}$ Alexander, ${ }^{1} \mathrm{~B}$ Arlett, ${ }^{1} \mathrm{SA}$ Bannister, ${ }^{1} \mathrm{TRK}$ Edwards, ${ }^{1} \mathrm{MT}$ Green, ${ }^{3} \mathrm{JDC}$ Ross, ${ }^{2} \mathrm{CA}$ Ison. ${ }^{1}$ Atlas Genetics; ${ }^{2}$ Public Health UK; ${ }^{3}$ University Hospitals Birmingham, UK

\subsection{6/sextrans-2015-052270.319}

Introduction The Atlas Genetics io $^{\mathrm{TM}}$ system is a new rapid molecular diagnostic platform designed to test for infectious diseases at Point of Care (POC). The test process is fully automated and utilises a novel nucleic acid detection technology. There is no specimen processing and it provides a result in 30 min. This preliminary evaluation compared the performance of the Atlas Genetics io ${ }^{\mathrm{TM}}$ Chlamydia trachomatis (CT) test to routine diagnostic testing using the APTIMA Combo 2 test (AC2, Hologic Gen-Probe) for the detection of CT.

Methods Two self-collected vulvo-vaginal swabs were obtained from women presenting at a genitourinary medicine clinic; swabs from alternate patients were placed in collection buffer and tested using the AC2 test or the io ${ }^{\mathrm{rM}} \mathrm{CT}$ assay as the first test. Any sample giving a discrepant result was retested using the residual buffer from the io ${ }^{\mathrm{TM}} \mathrm{CT}$ assay using the $\operatorname{artus}{ }^{\circledR} \mathrm{C}$. trachomatis Plus RG PCR kit (Qiagen). A true positive result was defined as positive with at least two of the three tests.

Results Of the samples collected from 193 women, 18 were determined to be true positive results for C. trachomatis, of which one sample was positive with the AC2 and artus CT test but negative with the io $^{\mathrm{TM}}$ CT test. Three io $^{\mathrm{TM}}$ false positive results were reported out of 175 samples that were negative when tested using the AC2 and artus tests. Based on a provisional cut-off, this resulted in a sensitivity and specificity of 94.4\% and 98.3\%, respectively for the io ${ }^{\mathrm{TM}}$ CT test.

Conclusion The Atlas Genetics io ${ }^{\mathrm{TM}}$ CT test was shown to deliver laboratory-equivalent results within $30 \mathrm{~min}$, on a system designed to be used in the STI clinic or similar POC setting, that is easy-to-use and gives results that require no interpretation or analysis.

Disclosure of interest statement BA, SAB, TRKE, MTG and DMP receive salaries and stock options from Atlas Genetics Ltd. This study was funded by the Technology Strategy Board, project No. 100845.

\section{P07.04 NEW MOLECULAR POINT-OF-CARE TEST IMPROVES TIMELINESS OF TREATMENT FOR CHLAMYDIA TRACHOMATIS (CT) AND NEISSERIA GONORRHOEA (NG) IN A REMOTE ABORIGINAL HEALTH CLINIC}

${ }^{1} R$ Huang* ${ }^{2}{ }^{2}$ W Ward ${ }^{*},{ }^{3} A$ Tangey, ${ }^{4} L$ Causer, ${ }^{4} R$ Guy, on behalf of the TTANGO investigators. ${ }^{1}$ Nganampa Health Council; ${ }^{2}$ South Australian Health and Medical Research Institute; ${ }^{3}$ Ngaanyatjarra Health Service; ${ }^{4}$ Kirby Institute

\subsection{6/sextrans-2015-052270.320}

Introduction High CT and NG prevalences have been observed in remote Aboriginal communities for decades. Testing and treatment are key prevention strategies, yet considerable delays in treatment occur, due to distances from laboratories and difficulties recalling patients. The TTANGO (Test, Treat ANd GO) randomised controlled trial is the first to evaluate whether a new point-of-care CT/NG test (GeneXpert) can improve the timelines of treatment and reduce re-infections rates in remote Aboriginal communities.

Methods In the context of TTANGO, we conducted an interim analysis to compare timelines of treatment before and after the point-of-care test was introduced at one of the 12 TTANGO sites. This site is one of seven remote clinics managed by Nganampa Health Council (NHC), an Aboriginal health service in South Australia.

Results Overall, 777 people were tested for CT/NG, 81 (10.4\%) were positive; highest in 15-19 year olds (15.4\%). In the pointof-care period, 40/40 (100\%, 95\% CI: 91.1-100) of people with a positive CT/NG point-of-care test received treatment, and of these $90 \%$ were treated in $24 \mathrm{~h}$, with a median time-to- 
treatment of 0 days. In the standard-care period, $40 / 41(97.6 \%$, 95\% CI: 87.1-99.9) received treatment; 15 (37.5\%) were treated presumptively on the same day (due to symptoms/risk) and $25(62.5 \%)$ were treated on the basis of the laboratory test result; and of these $44 \%$ were treated in 1-7 days, $44 \%$ in $8-14$ days and $12 \%$ in $15+$ days, with a median time-to-treatment of 8 days.

Conclusion This site is already part of a strong and comprehensive STI control program run by NHC over 20 years. Early findings from TTANGO show further improvement in STI management were achieved with point-of-care tests, with treatment occurring on average 8 days sooner for those treated on the basis of a test result. Future analyses will include all 12 clinics and also assess if re-infections have reduced.

Disclosure of interest statement No pharmaceutical grants were received in the development of this study. TTANGO is funded by a NHMRC project grant. The GeneXpert cartridges were purchased from Cepheid and Cepheid provided machines on loan for the duration of the study.

\section{P07.05 UTILITY OF POOLED URINE SAMPLES FOR DETECTION OF CHLAMYDIA TRACHOMATIS INFECTION IN ASYMPTOMATIC PREGNANT WOMEN IN NORTHERN INDIA}

${ }^{1} \mathrm{~S}$ Sethi*, ${ }^{1}$ A Roy, ${ }^{2}$ R Bagga, ${ }^{3}$ SD Mehta. ${ }^{1}$ Department of Medical Microbiology, General Hospital, Chandigarh; 'Department of Obstertics and Gynaecology, General Hospital, Chandigarh; ${ }^{3}$ Postgraduate Institute of Medical Education and Research Chandigarh, Department of Dermatology, General Hospital, Chandigarh

\subsection{6/sextrans-2015-052270.321}

Background Urogenital infections by Chlamydia trachomatis (CT) are the most prevalent sexually-transmitted bacterial diseases in women. Cost effective screening of women for C. trachomatis infection in developing countries is highly desirable for reducing morbidity and complications like pelvic inflammatory diseases, ectopic pregnancy and infertility. Results of noninvasive urine samples tests for C. trachomatis are nearly identical to samples collected directly from the cervix or urethra. The present study used the pooled urine samples to decrease the cost of screening for C. trachomatis.

Methods First void urine samples were collected from 1000 asymptomatic pregnant women having gestational age less than 24 weeks attending the Antenatal Clinics at PGIMER, Chandigarh during July 2009 to June 2012. The pooled urine samples (5x pooled processed specimen) were tested for presence of $C$. trachomatis by Amplicor CT PCR kit (Roche Diagnostic) and positive results were further tested separately on each urine sample. Direct fluroscent antibody test (DFA) assay was used on urine specimen which were positive by PCR to confirm the positive results.

Results Overall C. trachomatis infection tested by both PCR and DFA was present in $1.6 \%(16 / 1000)$ of asymptomatic pregnant women. A total of 200 pools of urine samples were tested and 20 pools were positive for C. trachomatis. When these pools were tested individually, 20 (10\%) samples were positive for CT (In one pool, 2 samples were positive and one pool was false positive). Four samples were negative by DFA. Pooling of urine samples saved $70 \%$ of reagent costs in our study.

Conclusions The study shows C. trachomatis infection was present in $1.6 \%$ of pregnant women which indicates low prevalence of infection in northern India. Pooling of urine samples saved labour, cost (70\% reduction) and time in screening large number of samples in resource-limited settings.

Disclosure of interest statement None (No conflict of interest). The study was funded by Indian Council of Medical Research, New Delhi. No pharmaceutical grants were received in the development of this study.

\section{P07.06 A LOW COST, HAND-HELD POINT OF CARE MOLECULAR DIAGNOSTIC DEVICE FOR SEXUALLY TRANSMITTED INFECTIONS}

${ }^{1}$ RE Mackay ${ }^{*},{ }^{1} M$ Branavan, ${ }^{1,2} \mathrm{P}$ Craw, ${ }^{1}$ A Naveenathayalan, ${ }^{3}$ ST Sadiq, ${ }^{1}$ W Balachandran. ${ }^{1}$ Brunel University London; ${ }^{2}$ Commonwealth Scientific and Industrial Research Organisation, Hobart; ${ }^{3}$ St. George's University of London

\subsection{6/sextrans-2015-052270.322}

Introduction Rapid and accurate field diagnostics have potential to impact on the burden of STIs in resource poor settings. Microfluidic and nano technologies offer opportunities to create molecular detection platforms but costs may be prohibitive. We present a low cost isothermal amplification, point of care test for rapid identification of sexually transmitted infections. Sample collection integrates directly with a microfluidic device for automated sample preparation, isothermal amplification and optical detection.

Methods Cell lysis, within the microfluidic cartridge, is conducted using a chemical method and nucleic acid purification is achieved on activated cellulose membrane. The microfluidic device incorporates passive mixing of lysis-binding buffers and sample using a serpentine channel. Isothermal amplification is conducted using thermophillic helicase dependent amplification (tHDA) and recombinase polymerase amplification (RPA). A low cost real-time isothermal amplification platform has been developed capable of running six amplifications simultaneously.

Results Results have shown extraction efficiencies for the new membrane of $69 \%$ and $57 \%$ compared to commercial Qiagen extraction of $85 \%$ and $59.4 \%$ for $0.1 \mathrm{ng} / \mu \mathrm{L}$ and $100 \mathrm{ng} / \mu \mathrm{L}$ salmon sperm DNA respectively spiked in phosphate buffered solution. Extraction experiments using the serpentine passive mixer cartridges incorporating lysis and nucleic acid purification showed extraction efficiency around $80 \%$ of the commercial Qiagen kit. The platform is capable of detecting $1.32 \times 10^{6}$ copies of target DNA through thermophillic helicase dependent amplification and $1 \times 10^{5}$ copies of Chlamydia trachomatis genomic DNA within 10 min through RPA.

Conclusion We have produced a low cost, rapid nucleic acid extraction, isothermal amplification and detection platform consistent with use remote resource poor settings. The simple optics setup demonstrated high sensitivity and rapid detection of the tHDA and RPA reactions removing the requirement for expensive dichroic filters and lenses. Diagnostic performance of the device is currently being undertaken.

Disclosure of interest statement No Disclosure of interest.

\section{P07.07 MULTIPLEXING STI CAUSING PATHOGENS USING MNAZYME QPCR: A NOVEL REAL-TIME TECHNOLOGY WITH A SUPERIOR CAPACITY FOR MULTIPLEXING}

SM Erskine, L Tan, S Walker, C Denver, E Mokany*. SpeeDx Pty Ltd, National Innovation Centre, Eveleigh, Australia

10.1136/sextrans-2015-052270.323 